\title{
Table of Units
}

Unless otherwise mentioned, in this book we will strictly adopt only SI units of measure for all quantities. Commonly used alternatives are the Lorentz-Heaviside units and Gaussian units; however, utilising these necessitates a formulation of the Maxwell equations different to that which is presented in this book.

Table 1: SI units of measure for bodies in three-dimensional space. Note that the quantities represented here have symbols presented for the spatial configuration. Alternative representations of certain units are shown in parentheses.

\begin{tabular}{|c|c|c|c|c|c|}
\hline Quantity & Symbol & SI unit & Quantity & Symbol & SI unit \\
\hline Magnetic quantities & & & Electric quantities & & \\
\hline $\begin{array}{l}\text { Current } \\
\text { Current element } \\
\text { Current density } \\
\text { Magnetic scalar potential } \\
\text { Magnetic vector potential } \\
\text { Magnetic field } \\
\text { Magnetic induction } \\
\text { Magnetisation }\end{array}$ & $\begin{array}{l}\dot{\mathrm{i}} \\
\mathrm{d} \dot{\mathrm{i}} \\
\dot{\mathrm{j}} \\
\Phi \\
\mathrm{a} \\
\mathrm{h} \\
\mathrm{b} \\
\mathrm{m}\end{array}$ & $\begin{array}{l}\mathrm{A}\left(\mathrm{Nm} \mathrm{V}^{-1} \mathrm{~s}\right) \\
\mathrm{Am}^{-1} \\
\mathrm{~A} \mathrm{~m}^{-2} \\
\mathrm{~A} \\
\mathrm{~T} \mathrm{~m} \\
\mathrm{~A} \mathrm{~m}^{-1} \\
\mathrm{~T}\left(\mathrm{~N} \mathrm{~A}^{-1} \mathrm{~m}\right) \\
\mathrm{A} \mathrm{m}^{-1}\end{array}$ & $\begin{array}{l}\text { Electric field } \\
\text { Dielectric displacement } \\
\text { Polarisation }\end{array}$ & $\begin{array}{l}\mathbb{e} \\
\mathbb{d} \\
\mathbb{p}\end{array}$ & $\begin{array}{l}\mathrm{V} \mathrm{m}^{-1} \\
\mathrm{Cm}^{-2} \\
\mathrm{Cm}^{-2}\end{array}$ \\
\hline \multicolumn{6}{|c|}{ Mechanical quantities } \\
\hline $\begin{array}{l}\text { Volume element } \\
\text { Area element } \\
\text { Mass density } \\
\text { Displacement } \\
\text { Velocity } \\
\text { Acceleration } \\
\text { Force }\end{array}$ & $\begin{array}{l}\text { dv } \\
\text { da } \\
\rho \\
\text { u } \\
\mathbf{v} \\
\text { a } \\
f\end{array}$ & $\begin{array}{l}\mathrm{m}^{3} \\
\mathrm{~m}^{2} \\
\mathrm{~kg} \mathrm{~m}^{-3} \\
\mathrm{~m} \\
\mathrm{~m} \mathrm{~s}^{-1} \\
\mathrm{~m} \mathrm{~s}^{-2} \\
\mathrm{~N}\end{array}$ & $\begin{array}{l}\text { Displacement gradient } \\
\text { Stress } \\
\text { Pressure } \\
\text { Body force density } \\
\text { Traction density } \\
\text { Moment density } \\
\text { Moment }\end{array}$ & $\begin{array}{l}\mathbf{F} \\
\boldsymbol{\sigma} \\
p \\
\mathbf{b} \\
\mathrm{t} \\
\mathrm{m} \\
\mathrm{m}\end{array}$ & $\begin{array}{l}- \\
\mathrm{Pa}\left(\mathrm{N} \mathrm{m}^{-2}\right) \\
\mathrm{Pa} \\
\mathrm{N} \mathrm{m}^{-3} \\
\mathrm{~N} \mathrm{~m}^{-2} \\
\mathrm{Nm}^{-2} \\
\mathrm{Nm}\end{array}$ \\
\hline \multicolumn{6}{|c|}{ Thermodynamic quantities } \\
\hline $\begin{array}{l}\text { Temperature } \\
\text { Energy }\end{array}$ & $\begin{array}{l}\theta \\
\Psi, \Omega\end{array}$ & $\begin{array}{l}\mathrm{K} \\
\mathrm{J}(\mathrm{N} \text { m) }\end{array}$ & $\begin{array}{l}\text { Entropy } \\
\text { Power }\end{array}$ & $\begin{array}{l}\eta \\
\mathscr{P}\end{array}$ & $\begin{array}{l}\mathrm{JK}^{-1} \\
\mathrm{~W}\left(\mathrm{~J} \mathrm{~s}^{-1}\right)\end{array}$ \\
\hline
\end{tabular}


